\title{
NuInt05 Session 2 Summary: New Experimental Results
}

\author{
L. Ludovici ${ }^{\mathrm{a}}$, K. S. McFarland ${ }^{\mathrm{b}}$, M. Shiozawa ${ }^{\mathrm{c}}$, G. P. Zeller ${ }^{\mathrm{d}}$ \\ ${ }^{a}$ University of Rome La Sapienza and INFN, I-000185 Rome, Italy \\ bUniversity of Rochester, Rochester, NY 14627 USA \\ 'Kamioka Observatory, Institute for Cosmic Ray Research, University of Tokyo, Kamioka, Gifu \\ 506-1205, Japan \\ ${ }^{\mathrm{d}}$ Columbia University, Department of Physics, New York, NY 10027 USA
}

\begin{abstract}
We summarize the discussion and presentation of new experimental results in low energy neutrino scattering at the NuInt05 workshop. In addition, we comment on several investigations that we would like to see targeted in preparation for the next NuInt workshop.
\end{abstract}

\section{New Experimental Results}

We were fortunate that all of the presentations in the NuInt05 second session showcased new or updated experimental results from a variety of experiments. In the case of charged current (CC) single $\pi^{+}$production, this included the first experimental measurement of $\mathrm{CC}$ coherent pion production at low energy in the K2K SciBar detector 1 and the first measurement of the ratio of $\mathrm{CC} 1 \pi^{+}$to $\mathrm{CC}$ quasi-elastic (QE) cross sections on a nuclear target at low energy from MiniBooNE 2]. NOMAD presented their new measurements of both QE and inclusive CC cross sections on carbon 3 . In addition, MINOS just started taking beam data a few months before the workshop and presented their first near detector neutrino event distributions 4]. In the neutral current $(\mathrm{NC})$ sector, $\mathrm{K} 2 \mathrm{~K}$ reported the first observation of neutrino-induced photons from nuclear de-excitation with the goal of measuring a NC elastic/QE cross section ratio on water [5]. The session ended with presentation of the first QE neutrino data in liquid argon from exposure of the small ICARUS prototype detector in the CERN WANF beam [6].

In many cases, these presentations added data in new energy regions or on new target materi- als. While we are clearly benefitting from this new data, these results certainly raise new questions. Many of these questions can be answered with data that is currently in hand or that will be collected in the near future. To ensure further progress and resolution of these questions, we present the issues and questions to address in the next NuInt workshop.

\section{Low $Q^{2}\left(Q^{2}<0.2 \mathbf{G e V}^{2}\right)$}

Since the beginning of the NuInt workshop series, we have seen significant and persistant disagreement at low $Q^{2}$ between model predictions and data from multiple experiments. This is perhaps not very surprising as this is the region where nuclear corrections are largest and most challenging to model.

Throughout the course of the NuInt workshops, we have learned that both the $\mathrm{K} 2 \mathrm{~K}$ and MiniBooNE data samples exhibit very similar $Q^{2}$ behavior; also, a larger low $Q^{2}$ deficit is observed in single pion than in QE data. We propose a detailed, quantitative comparison between the low $Q^{2}$ behavior in all of available $\mathrm{CC}$ exclusive samples on various targets and from multiple experiments. This includes comparisons between $\mathrm{QE}$, non-QE, and exclusive $\mathrm{CC} 1 \pi^{+}, \mathrm{CC} 1 \pi^{0}$, 
and $\mathrm{CC}$ multi- $\pi$ data. It would also be interesting to compare the $\mathrm{K} 2 \mathrm{~K}$ and MiniBooNE ${ }^{12} \mathrm{C}$ and ${ }^{16} \mathrm{O}$ data to data on heavier targets such as ${ }^{56} \mathrm{Fe}$ (MINOS) and ${ }^{207} \mathrm{~Pb}$ (CHORUS).

At this workshop, $\mathrm{K} 2 \mathrm{~K}$ presented new results [1] indicating that CC coherent $\pi^{+}$production cross secton calculations are much too large, hence explaining the observed deficit of data events at low $Q^{2}$. This analysis selects a coherent-rich sample by requiring events have low $Q^{2},<0.1 \mathrm{GeV}^{2}$, and by removing events consistent with QE kinematics or which have extra activity at the vertex. While evidence from the $\mathrm{K} 2 \mathrm{~K}$ analyses is compelling, it is important to definitively settle the low $Q^{2}$ issue with complementary data. This includes supporting evidence from other kinematic distributions (e.g., $\cos \theta_{\pi}$, and $t$ ) as well as cross-checks against CC $1 \pi^{0}$ data where there is no expected coherent scattering contribution. In addition, we propose an explicit search for CC coherent pion production in existing data from MiniBooNE, to be compared to the $\mathrm{K} 2 \mathrm{~K}$ results.

Another interesting avenue of investigation is to check, with present data, whether we can claim consistency between observed levels of coherent pion production in both of the $\mathrm{CC} \pi^{+}$and $\mathrm{NC}$ $\pi^{0}$ data samples or whether we are seeing differences between $\mathrm{NC}$ and $\mathrm{CC}$ coherent pion production. Finally, antineutrino coherent pion production is also of great interest because coherent pion production cross sections are predicted to be similar for neutrino and antineutrino scattering, and therefore such a deficit should be even more pronounced in antineutrino single pion data where resonance pion production backgrounds are smaller.

\section{QE Scattering}

While we are enjoying the arrival of new, higher statistics QE data from K2K, MiniBooNE, and NOMAD, is it really clear that we better understand QE scattering on nuclear targets as a result? At this workshop, it was pointed out that $\mathrm{K} 2 \mathrm{~K}$ and NOMAD are extracting significantly different axial-vector mass $\left(M_{A}\right)$ parameters from a dipole fit to the $\mathrm{QE}$ axial form factor. To assess how meaningful these differences are, it is imperative that we establish a common formalism with which to directly compare experimental results. With identical nuclear models and modern vector form factors [7], we need to reassess the range of $M_{A}$ values extracted from modern $\mathrm{QE}$ data on a variety of targets $\left({ }^{12} \mathrm{C},{ }^{16} \mathrm{O},{ }^{56} \mathrm{Fe},{ }^{40} \mathrm{Ar}\right)$. What impact do different levels of backgrounds, efficiencies, and selection biases have on these results? How do the results obtained from rate versus shape $\left(d \sigma / d Q^{2}\right)$ fits compare? What do similar fits to single pion distributions yield for $M_{A}$ ?

Can more information be added? Is there anything to be gained by refitting old neutrino data on heavy targets? What are the prospects for MiniBooNE antineutrino QE data and $M_{A}$ fits? Can similar fits be performed on ${ }^{56} \mathrm{Fe}$ at MINOS [4] or with liquid argon data 6]? The current state of affairs demands a concerted effort to reassess QE (and more specifically, axial-vector) parameters measured from modern heavy target data.

\section{New Data}

We also encourage the collection of new data sets. It is also important that we continue to build up our body of knowledge on low energy neutrino cross section by adding new experimental data. This new data may, of course, help clarify some of the questions being raised by the current datasets or have the potential to raise new ones. Specifically, CC $1 \pi^{0}$ data can aid in disentangling resonant and coherent pion production at low $Q^{2}$. Studies of coherent scattering at MINERvA [8] and theoretical studies 9] indicate that kinematic separation of resonant scattering from coherent scattering may be easier at higher energies. Samples of $\nu_{e} \mathrm{QE}$ interactions serve as a direct check of the predicted $d \sigma / d Q^{2}$ distributions in $\nu_{e}$ versus $\nu_{\mu}$ events. Also, any new information on antineutrino scattering and scattering from other nuclear targets at both higher and lower $A$ than carbon or oxygen are, of course, always welcome. 


\section{New Theoretical Models}

It is essential that we maintain a continued effort to pit modern neutrino data against modern theoretical models. Theories advance most quickly when confronted with data on an equal footing. At this workshop, we have seen many examples of new theoretical calculations that could help experimentalists move beyond their antiquated Fermi Gas and Rein-Sehgal based Monte Carlos. At the same time, experiments need actual code as well as guidance on how to implement new theoretical predictions in regions where they are valid. To move forward, we encourage continued communication between theorists and experimentalists toward this end.

\section{Conclusions}

We challenge the next NuInt workshop to explicitly target the questions being raised by current experimental data. Specifically:

- How well do we understand the axial-vector form factor contribution to QE and resonant scattering?

- Do we have a consistent framework to compare results from different experiments?

- Do we truly understand the behavior at low $Q^{2}$ in all of our data samples?

- Do we see any obstacles to incorporating new theoretical calculations into our experimental descriptions?

- Are there any other data samples that should be studied in addition to those that have been reported in this and previous NuInt workshops?

We hope that we can continue to make progress by isolating new data samples and working toward detailed comparisons between various experimental results. It is clear that we need to forge ahead on all fronts in order to fully clarify these remaining issues.

\section{Acknowledgements}

The Session 2 convenors sincerely thank Makoto Sakuda and Okayama University for graciously hosting this workshop. Intellectual discourse never blossoms more fruitfully than it did in Okayama in the inspiring presence of the perfect grapes.

\section{REFERENCES}

1. H. Tanaka, these proceedings.

2. M. O. Wascko, these proceedings.

3. R. Petti, these proceedings.

4. A. Belias, these proceedings.

5. J. Kameda, these proceedings.

6. A. Curioni, these proceedings.

7. R. Bradford, these proceedings.

8. K. McFarland, these proceedings.

9. E.A. Paschos, these proceedings. 